\title{
Critical Care and Emergency Medicine
}

CASE STUDY

\section{Extracorporeal Cardiopulmonary Resuscitation in a 36-Year-Old Man with Viral Myocarditis Who Survived Prolonged Cardiac Arrest and Bedside Decompressive Laparotomy Neurologically Intact}

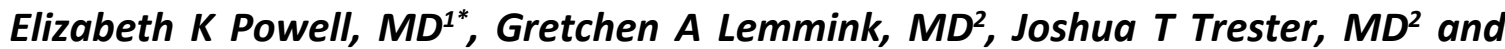 \\ Louis B Louis IV, MD
}

${ }^{1}$ Critical Care Fellow, Department of Anesthesiology, Division of Critical Care, University of Cincinnati, USA ${ }^{2}$ Assistant Professor, Department of Anesthesiology, Division of Critical Care, University of Cincinnati, USA

${ }^{3}$ Associate Professor, Department of Surgery, Division of Cardiothoracic Surgery, University of Cincinnati, USA

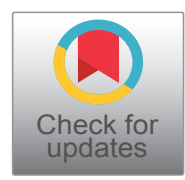

*Corresponding author: Elizabeth $K$ Powell, MD, Critical Care Fellow, Department of Anesthesiology, University of Cincinnati, 234 Goodman St, Cincinnati, OH 45219, USA, Tel: 484-614-3311

\section{Introduction}

We report a case of prolonged cardiac arrest in a patient with viral myocarditis who was subsequently cannulated for veno-arterial extracorporeal membrane oxygenation (VA-ECMO) while in cardiac arrest. The patient developed bowel ischemia and abdominal compartment syndrome on ECMO and required a bedside decompressive laparotomy and hemicolectomy. This case demonstrates that prolonged downtime with high quality CPR and potential ischemic complications of VA-ECMO should not preclude ongoing resuscitation and treatment as favorable neurologic outcome is still possible.

\section{Case Description}

The patient is a 36-year-old man with a history of hypertension and hyperlipidemia who originally presented to an outside hospital emergency department (ED) for evaluation of a syncopal episode. The patient was previously healthy until he experienced fevers and myalgias as well as nausea over the course of 48 hours. He had no shortness of breath, chest pain, abdominal pain, or any other complaints at the time of presentation. He had never previously had a syncopal episode. The patient had an initial ECG in the ED showing lateral
T wave inversions with no previous ECG for comparison. Lab work and imaging was performed and then while in the ED the patient developed chest pain. A repeat ECG demonstrated ST elevation in leads $\mathrm{V}_{2}-\mathrm{V}_{4}$ and his troponin returned at 7. The patient's BNP was 91 and he had no other laboratory abnormalities. A CXR showed no acute cardiopulmonary process. The patient was taken for a left heart catheterization (LHC) and had normal coronary arteries. He was admitted to the outside hospital intensive care unit with the diagnosis of non-ST elevation myocardial infarction.

The patient remained on room air overnight in the ICU for monitoring of his severe troponinemia. He did have a fever to 101.5F. Urine drug screen was negative. CT for pulmonary embolus was negative. A transthoracic echocardiogram (TTE) performed on day 1 of the patient's hospitalization revealed an EF of $40-45 \%$ with diffuse hypokinesis. Right ventricular function was normal.

On day 2 of hospital admission, the patient developed worsening shortness of breath and troponins continued to elevate. He continued to have intermittent fevers. Given the concern for myocarditis and worsening clinical status, the patient was transferred to the nearest tertiary referral center with advanced

Citation: Powell EK, Lemmink GA, Trester JT, Louis LB (2019) Extracorporeal Cardiopulmonary Resuscitation in a 36-Year-Old Man with Viral Myocarditis Who Survived Prolonged Cardiac Arrest and Bedside Decompressive Laparotomy Neurologically Intact. Int J Crit Care Emerg Med 5:087. doi. org/10.23937/2474-3674/1510087

Accepted: August 24, 2019: Published: August 26, 2019

Copyright: (c) 2019 Powell EK, et al. This is an open-access article distributed under the terms of the Creative Commons Attribution License, which permits unrestricted use, distribution, and reproduction in any medium, provided the original author and source are credited. 
heart failure capabilities. At approximately 03:50 on the third day of hospital admission the patient was transferred to the cardiovascular intensive care unit (CVICU). He arrived on non-invasive bilevel positive airway pressure ventilation with respiratory distress and was subsequently orotracheally intubated due to worsening hypoxia. The patient then developed ventricular tachycardia (VT) but initially with a pulse. A synchronized cardioversion was performed and the patient lost his pulse while still in VT necessitating defibrillation. The patient regained a pulse and was loaded with amiodarone and then lidocaine for refractory VT. He lost a pulse again and multiple rounds of CPR, defibrillation, epinephrine, calcium, and bicarbonate were administered. The patient was still in VT. Cardiac surgery was consulted for emergent veno-arterial extracorporeal membrane oxygenation (VA-ECMO) cannulation and access in the groin was obtained. A 10,000 unit heparin bolus was administered. An arterial cannula with antegrade perfusion was placed in the right femoral artery, and a venous cannula was placed in the left femoral vein. The patient achieved full flow on circuit 86 minutes after the last time he was pulseless and 105 minutes since initial cardiac arrest.

Hemodynamics stabilized shortly after initiation of VA-ECMO and the patient did require nitroglycerin for hypertension. A bedside TTE revealed no native cardiac activity. He was started on inotropic support. Approximately 12 hours after cannulation the patient was noted to be awake and following commands.

On hospital day 4 and ECMO day 1 the patient developed complete heart block and had a temporary pacer placed. He also had a right heart catheterization, and myocardial biopsy. Continuous renal replacement therapy (CRRT) was initiated for oliguric acute kidney injury.

On hospital day 5 (ECMO day 2) the patient's biopsy results revealed evidence of lymphocytic myocarditis with some eosinophils. All other viral and bacterial studies were negative. The patient was started on an immunosuppression regimen which consisted of steroids and mycophenolate and then cyclosporine. On the evening of hospital day 5 the patient was also noted to have a dusky right hand where the arterial line was placed. The arterial line was changed to the left radial artery. The patient also had evidence of ischemia to the bilateral lower extremities. Overnight, the patient developed increased abdominal distension without clinical evidence of bowel function. His PTT on a heparin infusion had been in the therapeutic range (60-80 seconds) since cannulation.

Given the abdominal distension that developed overnight as well as a lactic acidosis of $5 \mathrm{mmol} / \mathrm{L}$ and a creatine kinase (CK) as high as $20,000 \mathrm{U} / \mathrm{L}$, acute care surgery was consulted on hospital day 6 (ECMO day 3 ) for evaluation. There was concern for abdominal compartment syndrome secondary to an ischemic process, so the decision was made to proceed with bedside exploratory laparotomy (ex lap). The surgical team performed an emergent bedside decompressive laparotomy. The patient was found to have an ischemic right colon necessitating a right hemicolectomy with end ileostomy, retroperitoneal packing, and temporary abdominal closure. The patient's lactic acidosis and elevated CK improved dramatically, and his abdomen was closed the next day.

Multiple repeat TTEs were performed and the patient gradually regained cardiac function over the course of several weeks to a left ventricular ejection fraction of $50 \%$. His temporary pacemaker was removed. The patient was extubated while still on ECMO and was able to communicate with his family and appropriately answer questions. He moved all 4 extremities and had a Glasgow Coma Scale of 15. Physical and occupational therapy continued to work with him.

On hospital day 19 (ECMO day 16) the patient was decannulated by vascular surgery. The patient subsequently had bilateral below the knee guillotine amputations for gangrenous lower extremities thought to be secondary to his prolonged low flow state and subsequent ischemia.

The patient remained neurologically intact throughout his hospital stay. He was awake, alert, oriented, out of bed to chair, and evaluated by physical medicine and rehabilitation for prosthetics. He was discharged on hospital day 47 to a rehabilitation facility.

\section{Discussion}

In this case we report a patient who was discharged from the hospital neurologically intact following a 105-minute total arrest time and bedside decompressive laparotomy. The prolonged nature of the arrest with discharge neurologically intact and the bedside ex lap make this case unique.

With the adoption of extracorporeal cardiopulmonary resuscitation (eCPR), this type of support has proven an effective alternative for the support of adult patients following cardiac arrest, with survival rates ranging between $30 \%$ and $40 \%[1,2]$. Typically, the goal of eCPR initiation is within 60 minutes as survival dramatically decreases after this time [3]. Survival has been linked to duration of low flow state and time to eCPR [4]. Longer low flow times lead to decreased likelihood of recovery. In our case of in hospital cardiac arrest, high quality CPR until VA-ECMO was initiated likely significantly contributed to patient's neurologic outcome and suggests that high quality CPR initiated immediately at cardiac arrest can increase the time for VA-ECMO consideration and initiation.

In addition to the emergent bedside eCPR cannulation, the patient also had a bedside ex lap for increasing abdominal distention and concern for compart- 
ment syndrome and ischemia. There could be several etiologies for the patient's bowel ischemia. First, the prolonged downtime could have led to a primary low flow ischemic event [5]. Second, given the patient had no cardiac activity for one week after return of spontaneous circulation, a thrombus could have developed in the left ventricle which could have embolized $[6,7]$; however, our patient was heparinized with a therapeutic PTT, and no thrombus was ever seen on TTE.

Laparotomy for VA-ECMO patients is a rare event. Often, these patients are too hemodynamically unstable to be transported to the operating room thus necessitating bedside procedures. One study examined 7 total patients who had an ex lap while on VA-ECMO [8]. Five died. Similar to our patient, the first patient who survived had abdominal compartment syndrome and was found to have an ischemic small bowel. In our case, timely intervention probably allowed for prevention of further bowel necrosis and inflammatory response which would carry a high mortality.

Our case demonstrates several important VA-ECMO considerations. First, our patient had a favorable neurologic outcome with a prolonged low flow state with high quality CPR. In select patients, consideration for eCPR cannulation after witnessed prolonged downtime, suspected reversible etiology, and high-quality CPR should be considered. Second, early recognition of abdominal compartment syndrome and decompressive laparotomy due to high suspicion of ischemic event allowed for patient recovery. Though associated with a high mortality, bedside laparotomy can be performed in the VA-ECMO patient population.

\section{References}

1. Combes A, Leprince P, Luyt CE, Bonnet N, Trouillet JL, et al. (2008) Outcomes and long-term quality-of-life of patients supported by extracorporeal membrane oxygenation for refractory cardiogenic shock. J Crit Care Med 36: 14041411.

2. Jaski BE, Ortiz B, Alla KR, Smith SC Jr, Glaser D, et al. (2010) A 20-year experience with urgent percutaneous cardiopulmonary bypass for salvage of potential survivors of refractory cardiovascular collapse. J Thorac Cardiovasc Surg 139: 753-757.

3. Conrad SA, Rycus P (2017) Extracorporeal membrane oxygenation for refractory cardiac arrest. Ann Card Anaesth 20: $4-10$

4. Wengenmayer $\mathrm{T}$, Rombach $\mathrm{S}$, Ramshorn $\mathrm{F}$, Biever $\mathrm{P}$, Bode C, et al. (2017) Influence of low-flow time on survival after extracorporeal cardiopulmonary resuscitation (eCPR). Crit Care 21: 157.

5. Korth U, Krieter H, Denz C, Janke C, Ellinger K, et al. (2003) Intestinal ischaemia during cardiac arrest and resuscitation: Comparative analysis of extracellular metabolites by microdialysis. Resuscitation 58: 209-217.

6. Makdisi G, Hashmi ZA, Wozniak TC, Wang IW (2015) Left ventricular thrombus associated with arteriovenous extra corporeal membrane oxygenation. J Thorac Dis 7: E552-E554.

7. Gaide-Chevronnay L, Durand M, Rossi-Blancher M, Bach V, Chavanon O, et al. (2012) Cardiac thrombosis in a patient during extracorporeal life support. J Cardiothorac Vasc Anesth 26: 664-665.

8. Sakata KK, Kashyap R, Guru PK, Seelhammer T, Barbara $D$, et al. (2016) Outcomes of patients on VA ECMO who underwent an exploratory laparotomy. American Journal of Respiratory and Critical Care Medicine 193: 7024. 